\title{
Early diagnosis and appropriate respiratory support for Mycoplasma pneumoniae pneumonia associated acute respiratory distress syndrome in young and adult patients: a case series from two centers
}

\author{
Lin Ding ${ }^{1 \dagger}$, Yu Zhao ${ }^{2 \dagger}$, Xuyan $\mathrm{Li}^{2}$, Rui Wang ${ }^{2}$, Ying $\mathrm{Li}^{2}$, Xiao Tang ${ }^{2}$, Bing $\mathrm{Sun}^{2 \dagger}$ and Hangyong $\mathrm{He}^{2^{*}}$ (D)
}

\begin{abstract}
Background: Mycoplasma pneumoniae (M. pneumoniae) is one of the most common causes of community acquired pneumonia (CAP). Establishing an early diagnosis of M. pneumoniae pneumonia in patients with acute respiratory distress syndrome (ARDS) may have important therapeutic implications.

Methods: We describe diagnosis and management of $M$. pneumoniae pneumonia induced ARDS in a case series of adults and youth hospitalized with radiographically confirmed CAP prospectively enrolled in an observational cohort study in two university teaching hospitals, from November 2017 to October 2019.

Results: In all 10 patients, early and rapid diagnosis for severe M. pneumoniae pneumonia with ARDS was achieved with polymerase chain reaction (PCR) or metagenomic next-generation sequencing (mNGS) testing of samples from the lower respiratory tract or pleural effusion. The average $\mathrm{PaO}_{2} / \mathrm{FiO}_{2}$ of all patients was $180 \mathrm{mmHg}$. Of the 10 cases, 4 cases had moderate ARDS $\left(100 \mathrm{mmHg} \leq \mathrm{PaO}_{2} / \mathrm{FiO}_{2}<200 \mathrm{mmHg}\right)$ and 3 cases had severe ARDS $\left(\mathrm{PaO}_{2} / \mathrm{FiO}_{2}<100\right.$ $\mathrm{mmHg}$ ). High flow nasal cannula (HFNC) was applied in all patients, though only two patients were sufficiently supported with HFNC. Invasive mechanical ventilation (IMV) was required in 5 patients. High resistance (median 15 $\mathrm{L} / \mathrm{cmH}_{2} \mathrm{O} / \mathrm{s}$ ) and low compliance (median $38 \mathrm{ml} / \mathrm{cmH}_{2} \mathrm{O}$ ) was observed in 4 cases. In these 4 cases, recruitment maneuvers (RM) were applied, with 1 patient demonstrating no response to RM. Prone positioning were applied in 4 cases. Two cases needed ECMO support with median support duration of 5.5 days. No patient in our case series received corticosteroid therapy. All patients were survived and were discharged from hospital.
\end{abstract}

\footnotetext{
* Correspondence: yonghang2004@sina.com

${ }^{\dagger}$ Lin Ding, Yu Zhao and Bing Sun contributed equally to this work.

${ }^{2}$ Department of Respiratory and Critical Care Medicine, Beijing Institute of Respiratory Medicine, Beijing Key Laboratory of Respiratory and Pulmonary Circulation, Beijing Chao-Yang Hospital, Capital Medical University, No. 8 Gongren Tiyuchang Nanlu, Chaoyang District, Beijing 100020, China Full list of author information is available at the end of the article
}

(c) The Author(s). 2020 Open Access This article is licensed under a Creative Commons Attribution 4.0 International License, which permits use, sharing, adaptation, distribution and reproduction in any medium or format, as long as you give appropriate credit to the original author(s) and the source, provide a link to the Creative Commons licence, and indicate if changes were made. The images or other third party material in this article are included in the article's Creative Commons licence, unless indicated otherwise in a credit line to the material. If material is not included in the article's Creative Commons licence and your intended use is not permitted by statutory regulation or exceeds the permitted use, you will need to obtain permission directly from the copyright holder. To view a copy of this licence, visit http://creativecommons.org/licenses/by/4.0/ The Creative Commons Public Domain Dedication waiver (http://creativecommons.org/publicdomain/zero/1.0/) applies to the data made available in this article, unless otherwise stated in a credit line to the data. 


\begin{abstract}
(Continued from previous page)
Conclusions: Early and rapid diagnosis of severe M. pneumoniae pneumonia with ARDS can be achieved with PCR/ mNGS tests in samples from the lower respiratory tract or pleural effusion. In our case series, half of $M$. pneumoniae pneumonia induced ARDS cases were adequately supported with HFNC or NIV, while half of cases required intubation. RM and prone position were effective in 30\% of intubated cases, and 20\% needed ECMO support. When early anti-mycoplasmal antibiotics were given together with sufficient respiratory support, the survival rate was high with no need for corticosteroid use.
\end{abstract}

Keywords: Mycoplasma pneumoniae(M. pneumoniae), Acute respiratory distress syndrome (ARDS), Communityacquired pneumonia (CAP), High flow nasal cannula (HFNC), Extracorporeal membrane oxygenation (ECMO)

\section{Background}

Mycoplasma pneumoniae (M. pneumoniae) is one of the most common causes of community acquired pneumonia (CAP) often seen in children and young adults, and accounts for $10-39 \%$ of all cases of adult CAP cases $[1,2]$. M. pneumoniae pneumonia is typically mild and characterized by a persistent dry cough or self-limiting pneumonia that resolves with no medication [3]. However, respiratory failure and severe acute respiratory distress syndrome (ARDS) occur in $0.5-2 \%$ of all $M$. pneumoniae pneumonia cases and primarily affect young adults [4-18]. The rates of intensive care unit (ICU) admission of hospitalized M. pneumoniae pneumonia patients are reported as $10 \%$ in the US and $16.3 \%$ in Europe $[19,20]$. The rate of ICU admission is even higher at $38.8 \%$ in patients older than 65 years, compared to $18 \%$ in patients older than 19 years [20]. In one retrospective study from our hospital, $4.1 \%$ of M. pneumoniae pneumonia patients needed ICU admission for acute respiratory failure in the setting of an epidemic [21].

Severe ARDS and fatal outcome as a result of $M$. pneumoniae pneumonia may be the result of unclear clinical features [5], delayed diagnosis, inappropriate respiratory support, and/or insufficient initial treatment. When acute nonbacterial pneumonia progresses, $M$. pneumoniae must be considered as a possible cause, and appropriate diagnosis, respiratory support and therapeutic measures should be promptly instituted. Previous studies suggest that $M$. pneumoniae infection should be included in the differential diagnosis of ARDS, and that establishing an early diagnosis may have important therapeutic implications [22].

In recent years, rapid diagnostic methods have been developed, allowing for early diagnosis of M. pneumoniae pneumonia. Detection of $M$. pneumoniae using fluorescence-quantatitive PCR in respiratory samples $[19,21-24]$ and metagenomic next-generation sequencing (mNGS) has increased [25]; these methods are especially useful for early detection of rare, atypical, and slow-growing microbes. Case reports have also described using new forms of respiratory support for $M$. pneumoniae pneumonia induced ARDS, such as highflow nasal cannula (HFNC) [26], non-invasive ventilation (NIV) [27] and veno-venous extracorporeal membrane oxygenation (ECMO) [18, 24, 28]. However, there has not yet been a full evaluation of the new available diagnostic and therapeutic measures in $M$. pneumoniae pneumonia induced ARDS.

The aim of our study was to describe a case series of 10 patients with $M$. pneumoniae pneumonia induced ARDS and provide an overview of available modalities for diagnosis and treatment. We describe the epidemiological, clinical, imaging, and laboratory features of our patients, review the available procedures for early diagnosis, and evaluate available respiratory support techniques in clinical practice in order to highlight the importance of rapid recognition and appropriate treatment.

\section{Methods}

\section{Study population}

We retrospectively identified all cases of young and adult patients (age over 14 years) with ARDS caused by $M$. pneumoniae pneumonia who were admitted to the respiratory ICU in two teaching hospitals (Beijing ChaoYang Hospital and Beijing Luhe Hospital, Beijing, China) with radiographically confirmed CAP from November 2017 to October 2019. The study was approved by the institutional review boards (IRB) at each institution. Written informed consent was obtained from all participants, where participants are children (under 16 years old) from their parent or guardian.

\section{Case definitions for M. pneumoniae pneumonia induced ARDS}

Criteria for diagnosis of $M$. pneumoniae pneumonia were based on 1) clinical signs and symptoms (cough, fever, productive sputum, dyspnoea, chest pain or abnormal breath sounds); 2) radiographic pulmonary abnormalities that were at least segmental and were not explained by pre-existing or other known causes; and 3) positive detection of $M$. pneumoniae nucleic acid by PCR or mNGS from lower respiratory tract secretion 
(sputum and BALF), which were considered as microbiological evidence of infection.

All cases of pneumonia occurring more than 3 days after hospitalization were considered nosocomial and were excluded. Patients with HIV infection, neutropenia, or who were receiving immunosuppressive chemotherapy were excluded.

The diagnosis of ARDS was assigned to patients who met the Berlin definition criteria: 1) presence of acute hypoxemic respiratory failure; 2) onset within 7 days of insult, or new (within 7 days) or worsening respiratory symptoms; 3) bilateral opacities on chest $\mathrm{x}$-ray or CT not fully explained by effusions, lobar or lung collapse, or nodules; and 4) cardiac failure not the primary cause of acute respiratory failure. We categorized patients into 3 mutually exclusive classes of ARDS severity using previous definitions based on degree of hypoxemia: 1) mild (200 mmHg $\leq \mathrm{PaO}_{2} / \mathrm{FIO}_{2}<300 \mathrm{mmHg}$ ); 2) moderate $\left(100 \mathrm{mmHg} \leq \mathrm{PaO}_{2} / \mathrm{FIO}_{2}<200 \mathrm{mmHg}\right) ;$ and 3) severe $\left(\mathrm{PaO}_{2} / \mathrm{FIO}_{2}<100 \mathrm{mmHg}\right)$.

\section{Microbiological tests}

Patient specimens, including sputum, whole blood, and serum samples, were collected upon admission and during hospitalization for bacterial and viral testing. Microbiological tests were performed at the Department of Infectious Disease and Clinical Microbiology laboratories in our centers. Throat swabs, sputum, endotracheal aspiration or BALF were collected for $M$. pneumoniae PCR assay. All severe ARDS patients had M. pneumoniae infection confirmed by PCR assay or mNGS from lower respiratory tract secretion (sputum and BALF). PCR of pleural effusion fluid were also tested in some patients for the detection of M. pneumoniae.

\section{Data collection}

Clinical information collected included the following: characteristics (age and sex), comorbidities, clinical symptoms (fever, cough, sputum, dyspnea, chest pain, rash, nausea, vomiting, abdominal pain, diarrhea and headache), clinical signs (body temperature, heart rate, respiratory frequency, blood pressure and crackles in the lungs), laboratory tests (whole-blood cell count and blood chemistry), and microbiological findings and images of the lung, including chest X-ray (CXR) and high resolution computed tomography (HRCT). Concomitant medications, respiratory support (HFNC, NIV, invasive mechanical ventilation, prone position and ECMO), complications, and outcomes were also recorded.

\section{Statistical analysis}

Pooled epidemiological, clinical, imaging, and laboratory data are shown as median with range for quantitative variables and as absolute and relative frequencies for qualitative variables. The enrolled patients were divided into two groups based on use of invasive mechanical ventilation. Continuous variables were compared using the Mann-Whitney U-test, whereas categorical data were compared using the Chi-squared test or the Fisher's Exact test, where appropriate. All comparisons were performed using the SPSS statistics package version 19.0. Differences were considered statistically significant when $p$ was $<0.05$.

\section{Results}

Baseline characteristics of the included patients

Between November 2017 and October 2019, 11 patients met criteria of severe $M$. pneumoniae pneumonia. Of the 11 patients, one patient was excluded due to diagnosis of lymphoma combined with adenovirus pneumonia. Therefore, 10 immunocompetent patients were included in the final analysis.

The age range of our patients was 15 to 53 (median 31) years. There were 6 male and 4 female patients. All cases were admitted in different months of the year except for January, February and September. Only one patient (case 9) had diabetes mellitus. The other 9 patients had no underlying diseases (Table 1).

\section{Microbiological findings}

Seven (70\%) and $5(50 \%)$ patients had positive serum $M$. pneumoniae IgG and IgM, respectively. M. pneumoniae PCR of the sputum was performed in $9(90 \%)$ cases, and was positive in all 9 cases. Three of the 9 cases had $M$. pneumoniae PCR from BALF at the same time, and all 3 cases (100\%) were positive. Another patient was diagnosed with M. pneumoniae pneumonia through PCR of pleural effusion fluid. Five cases had mNGS from BALF, and all these 5 cases were positive for $M$. pneumoniae (Table 1).

Acinetobacter baumannii was detected in 2 patients (20\%) who were transferred from another hospital after ICU admission, but these were isolated from the lower respiratory tract (LRT) samples collected after more than 3 days of their ICU stay, and therefore were not considered as causative agents of ARDS together with M. pneumoniae (Table 1).

\section{Clinical features of symptoms and signs, laboratory tests and radiologic findings Symptoms and signs}

All 10 patients had cough and fever at the onset of illness. They presented with a high fever, with a median body temperature of $40.3^{\circ} \mathrm{C}$ (range, $39.6{ }^{\circ} \mathrm{C}$ to $42.0^{\circ} \mathrm{C}$ ). Eight patients (80\%) had dry cough and two patients had productive cough. Four patients (40\%) had diarrhea and one patient (10\%) had abdominal pain (Table 1). Acute respiratory deterioration occurred 3 to 15 (median 9) 


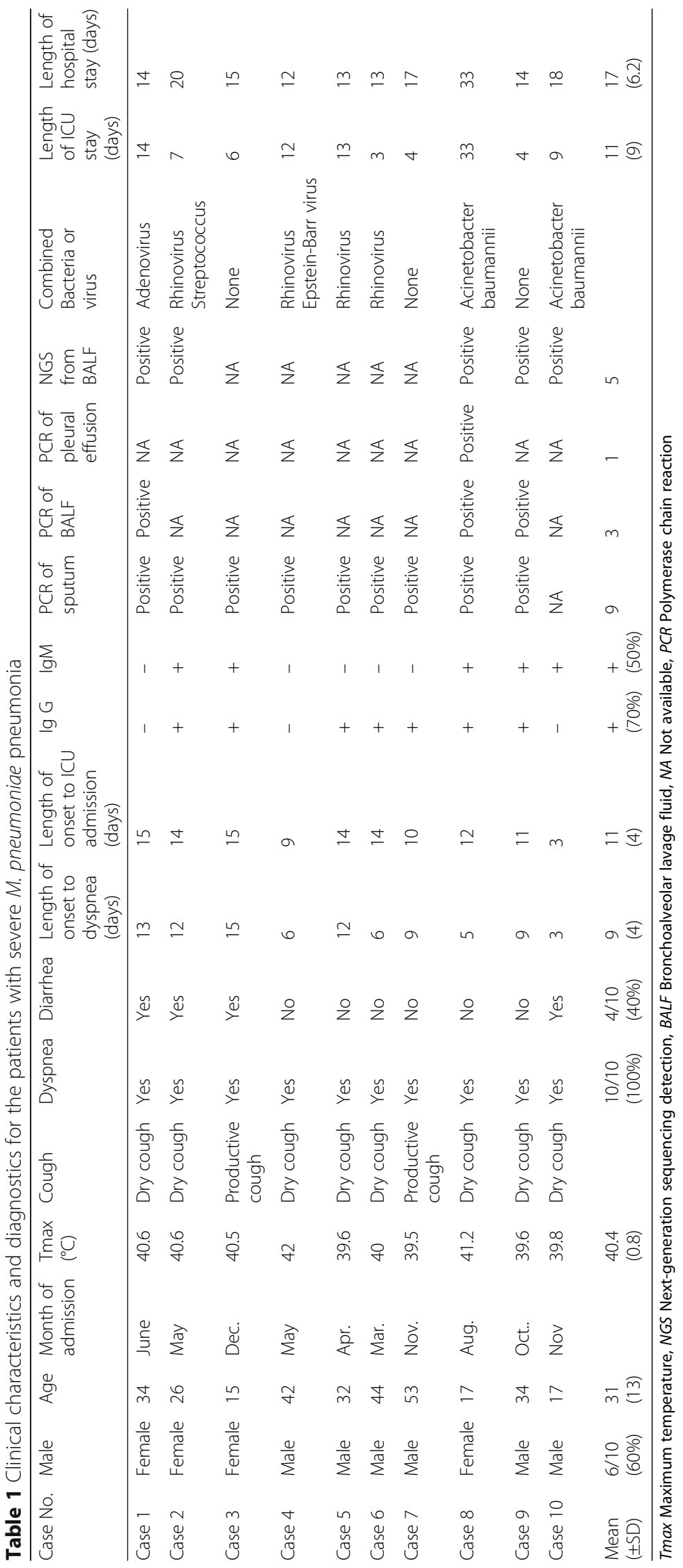


days after the onset of symptoms. After the onset of dyspnea, patients usually progressed to acute respiratory failure. The mean time from symptom onset to ICU admission was 11 days (range, 3 to 15 days) (Table 1). All 10 cases had tachypnea when admitted to the ICU, with mean respiratory rate of 27 breaths per minute (range, 21 to 33$)$.

\section{Laboratory tests}

Arterial blood gas analysis at ICU admission of all patients revealed hypoxia, with a mean $\mathrm{PaO}_{2} / \mathrm{FiO}_{2}$ of 180 (range $=47$ to 263 ) $\mathrm{mmHg}$. White blood cell counts were low or in the normal range on the first day of admission. All patients had elevated serum aspartate aminotransferase (AST), lactate dehydrogenase (LDH) and hydroxybutyrate dehydrogenase (HBDH). Eight patients $(80 \%)$ had elevated AST (44 U/L to $134 \mathrm{U} / \mathrm{L})$. Four patients (40\%) had elevated CK (457 U/L to $1052 \mathrm{U} / \mathrm{L})$. Nine patients (90\%) had elevated LDH and HBDH (328 U/L to $920 \mathrm{U} /$ $\mathrm{L}$, and $196 \mathrm{U} / \mathrm{L}$ to $637 \mathrm{U} / \mathrm{L}$ ) (Attached file 1, E-Table 1). Nine patients were tested for cell-mediated immunity, immunoglobulins (serum IgG, IgA and IgM), and components (Attached file 2, E-Table 2).

\section{Characteristics of pleural effusions}

In our study, eight patients had unilateral pleural effusions, and only one patient had bilateral pleural effusions. Pleural effusion fluid was examined in 4 patients. The pleural effusion fluid was light yellow and clear in 2 patients, and were yellow but turbid in the other 2 patients. Routine pleural effusion tests demonstrated mononuclear cell dominant leukocyte populations (Attached file 3, E-Table 3).

\section{Radiologic findings}

All 10 patients had CXRs. CXRs revealed bilateral multilobular or segmental consolidation in nine $(90 \%)$ patients. One patient's CXR showed diffuse peribronchial infiltration. All 10 patients underwent chest HRCT. Unilateral or bilateral consolidation and infiltration were found on HRCT scans of 9 patients (90\%). Large areas of consolidation within a single lobe or several lobes (90\%), followed by pleural effusion (80\%), were the most common findings on HRCT (Fig. 1). Only the HRCT of case 9 showed peribronchial infiltration without consolidation and pleural effusion.

\section{Respiratory mechanics and respiratory support}

The average $\mathrm{PaO}_{2} / \mathrm{FiO}_{2}$ of all patients was $180 \mathrm{mmHg}$. Four $(40 \%)$ cases had moderate ARDS $(100 \mathrm{mmHg} \leq$ $\mathrm{PaO}_{2} / \mathrm{FiO}_{2}<200 \mathrm{mmHg}$ ), and three cases (30\%) had severe ARDS $\left(\mathrm{PaO}_{2} / \mathrm{FiO}_{2}<100 \mathrm{mmHg}\right)$. HFNC was applied in all patients (100\%), with a median gas flow of $50 \mathrm{~L} / \mathrm{min}(45-53 \mathrm{~L} / \mathrm{min})$ and $\mathrm{FiO}_{2}$ 0.48(0.35-0.5), but only two patients were sufficiently supported with HFNC. NIV was used in four patients with a median duration of 24(13-32) h, and one patient had NIV failure and needed intubation (Table 2).

Invasive mechanical ventilation (IMV) was carried out in 5 patients (50\%). High airway resistance (median 15 $\mathrm{L} / \mathrm{cmH}_{2} \mathrm{O} / \mathrm{s}$ ) and low respiratory system compliance (median $38 \mathrm{ml} / \mathrm{cmH}_{2} \mathrm{O}$ ) was observed in all 5 cases. Recruitment maneuver (RM) was applied in 4 cases, with 1 patient not responsive to $\mathrm{RM}$, and 4 cases were put into prone position. The maximum PEEP was $14 \mathrm{cmH}_{2} \mathrm{O}$ (range, 11-16 $\mathrm{cmH}_{2} \mathrm{O}$ ). Two cases (20\%) needed ECMO support with median support duration of 5.5 days (Table 2). As shown in Table 2, Case 8 had the worst compliance and was unresponsive to RM, and ECMO was established. Case 1, case 3, and case 5 were responsive to RM and prone position.

\section{Treatment}

All 10 patients did not receive fluoroquinolones at the onset of their illness, and switched to fluoroquinolones $8.7 \pm 3.5$ days after onset. All patients received $\beta$-lactams treatment as first therapy, and 5 patients received treatment combined with macrolides before their admission to ICU. After ICU admission, moxifloxacin were given to all 10 patients.

No patients in our case series received corticosteroid therapy.

\section{Outcomes}

All patients survived in the ICU and were discharged from the hospital. The mortality of our cases was $0 \%$. The average length of ICU stay was 11 days, and the average length of hospital stay was 17 days.

\section{Comparison of intubated and non-intubated cases in MPP patients}

As shown in Table 3, compared to non-intubated patients, patients in intubated group were younger, were less likely to be male, had lower $\mathrm{PaO}_{2} / \mathrm{FiO}_{2}$ and higher APACHE II scores, and had higher procalcitonin and neutrophil proportion at ICU admission.

\section{Discussion}

To our knowledge, our study is the first and largest case series to evaluate diagnostic and therapeutic modalities in severe $M$. pneumoniae pneumonia induced ARDS. Our main findings are as follows: 1) early and rapid diagnosis for severe $M$. pneumoniae pneumonia with ARDS was achieved with PCR/mNGS test of samples from the lower respiratory tract and pleural effusions; 2) CT findings mainly showed alveolar patterns with bilateral consolidations rather than interstitial patterns; 3) respiratory mechanics showed low 


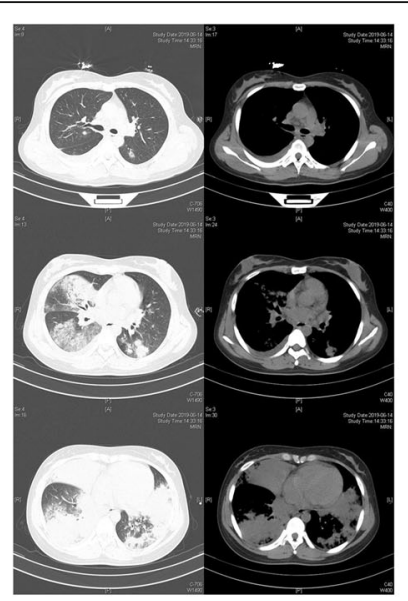

Case 1, Day 1

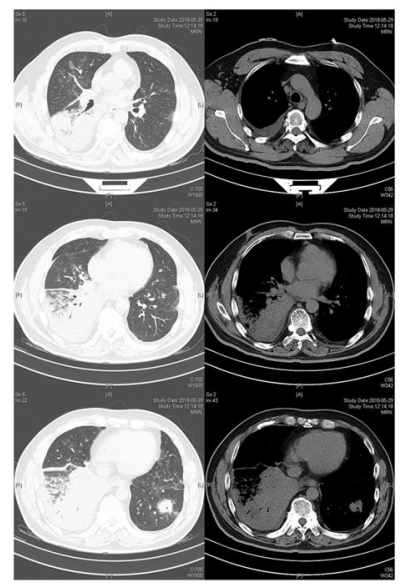

Case 4, Day 1

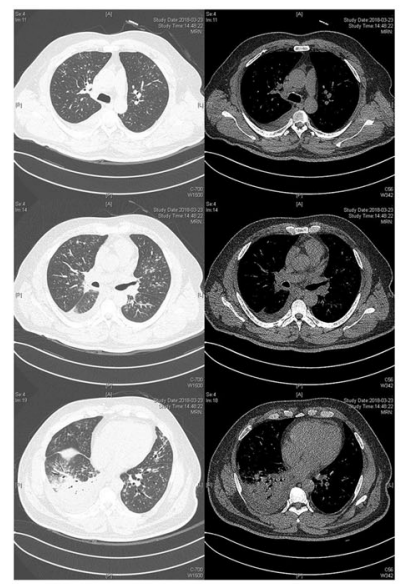

Case 6, Day 1

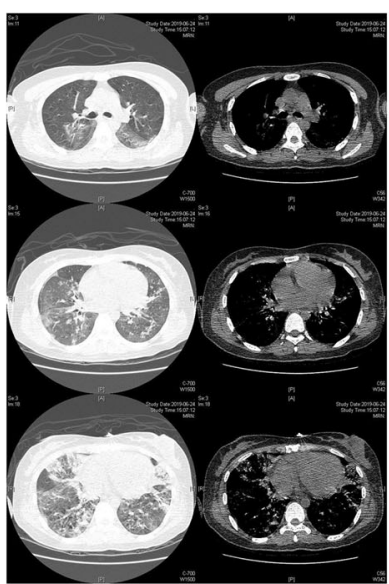

Case 1, Day 11

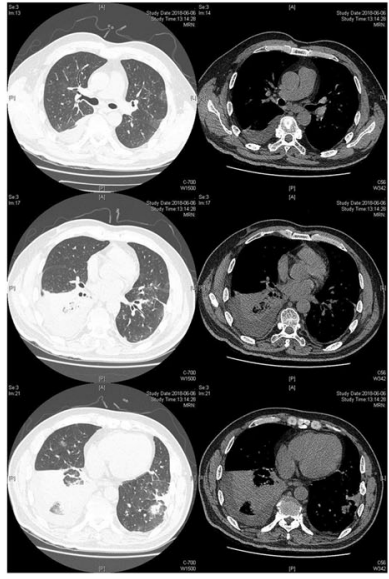

Case 4, Day 8

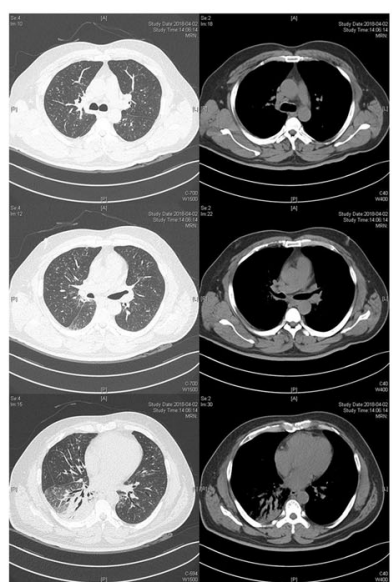

Case 6, Day 10

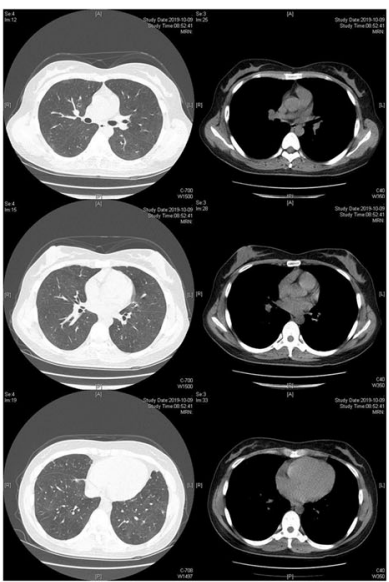

Case 1, 3 months

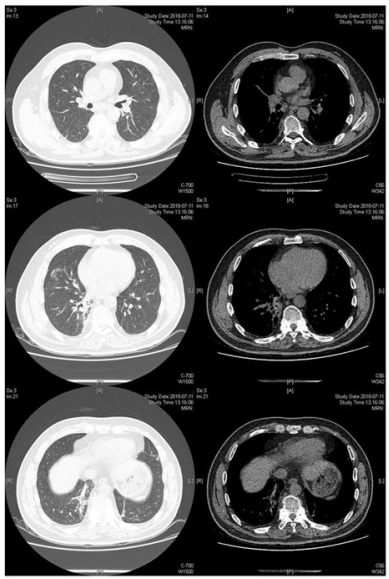

Case 4,1 months

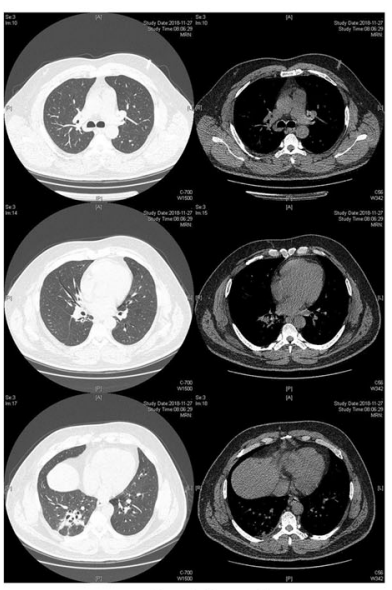

Case 6, 8 months

Fig. 1 Chest computed tomography (CT) on day 1, day 8-11, followed up after 1 months to 8 months of three patients (case1, case 4 and case 6). Three representative slices of the upper, middle and lower lobe were chosen

respiratory system compliance and high airway resistance; 4) $50 \%$ of M. pneumoniae induced ARDS were adequately supported with HFNC or NIV, $50 \%$ required intubation, RM and prone position were effective in 30\% intubated cases, and 20\% needed ECMO support; 5) when early anti-mycoplasmal drugs together with sufficient respiratory support are given, the survival rate was high with no need for corticosteroids; and 6) younger patients with lower $\mathrm{PaO}_{2} /$ $\mathrm{FiO}_{2}$ and APACHE II scores, and higher PCT and higher neutrophil cell proportion at ICU admission were more likely to require intubation. 


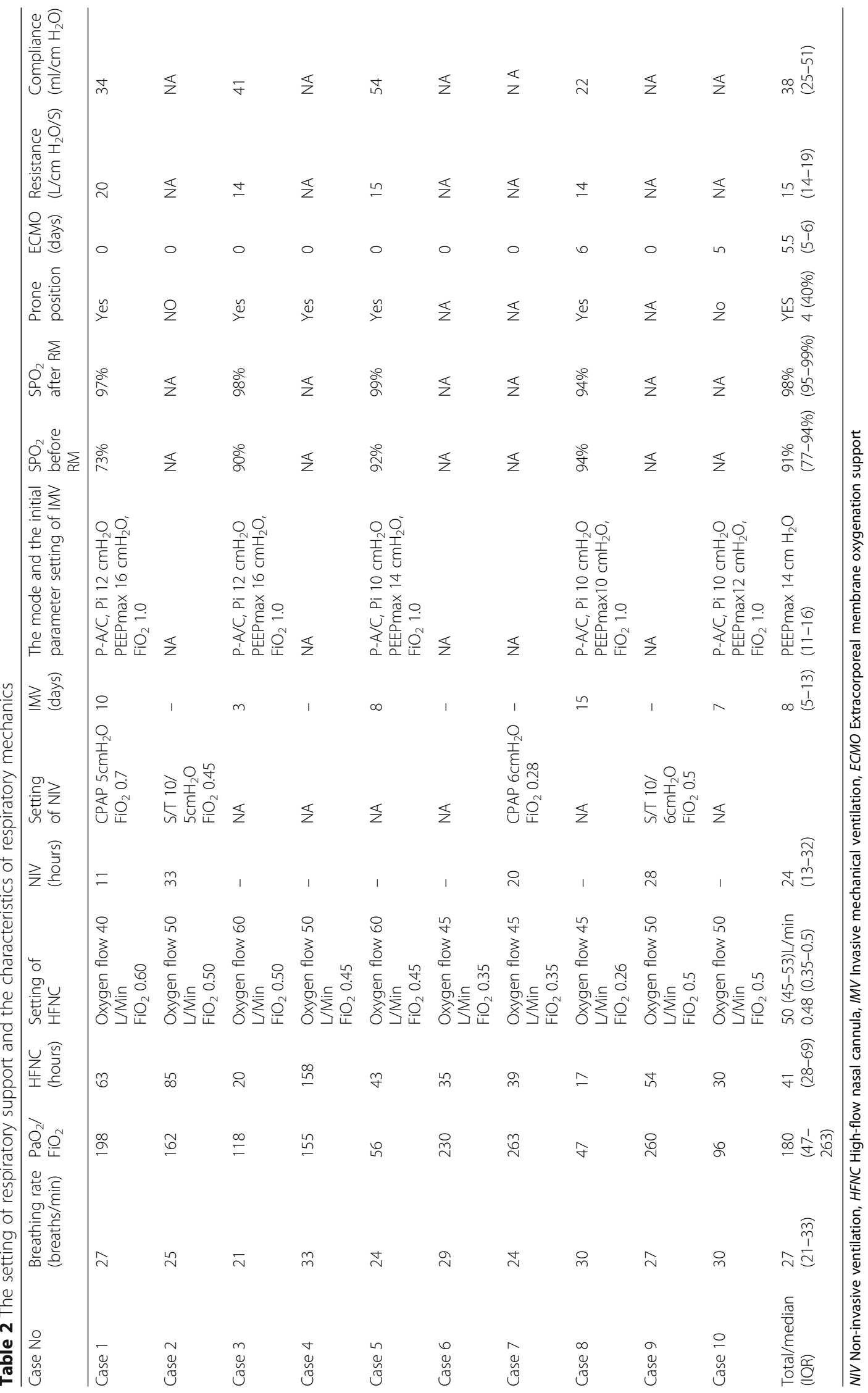


Table 3 Comparisons between patients with IMV and non-IMV (Median, IQR)

\begin{tabular}{|c|c|c|c|}
\hline & $\begin{array}{l}\text { Non-intubated group } \\
n=5\end{array}$ & $\begin{array}{l}\text { IMV group } \\
n=5\end{array}$ & $P$ value \\
\hline Male(n,\%) & $4(80 \%)$ & $2(40 \%)$ & $0.003^{*}$ \\
\hline Age (year) & $42(30-49)$ & $17(16-33)$ & $0.032^{*}$ \\
\hline APACHEII & $6(4-12)$ & $17(11-21)$ & $0.028^{*}$ \\
\hline $\mathrm{PaO}_{2} / \mathrm{FiO}_{2}$ & $230(159-262)$ & $96(51-158)$ & $0.032^{*}$ \\
\hline Lactate & $0.9(0.7-1.3)$ & $1.3(1.2-1.7)$ & 0.056 \\
\hline Breathing rate (breaths/min) & $26(25-31)$ & $27(23-29)$ & 0.690 \\
\hline $\operatorname{Tmax}\left(\left({ }^{\circ} \mathrm{C}\right)\right)$ & $40(39.5-41.3)$ & $40(39.7-40.9)$ & 0.841 \\
\hline Procalcitonin & $0.175(0.14-0.195)$ & $1.05(0.45-2.09)$ & $0.016^{*}$ \\
\hline White blood cell count $\left(10^{9} / \mathrm{L}\right)$ & $4.5(3.8-7.3)$ & $7.6(5.5-8.7)$ & 0.151 \\
\hline Neutrophil proportion(\%) & $73(54-82)$ & $92(83-94)$ & $0.016^{*}$ \\
\hline AST $(U / L)$ & $81(33-102)$ & $100(41-121)$ & 0.548 \\
\hline Albumin (g/L) & $29(28-34)$ & $32(26-39)$ & 1.0 \\
\hline Pre-albumin (g/L) & $0.08(0.02-0.15)$ & $0.03(0.03-0.04)$ & 0.571 \\
\hline Creatine (umol/L) & $55(47-69)$ & $43(35-76)$ & 1.0 \\
\hline C-reactive protein (CRP, mg/L) & $17(6-48)$ & $93(11-209)$ & 0.286 \\
\hline Erythrocyte sedimentation rate $(\mathrm{ESR}, \mathrm{mm} / \mathrm{h})$ & $25(5.5-28.5)$ & $12(4-25)$ & 0.675 \\
\hline Length of onset to ICU admission (days) & $11(9.5-14)$ & $14(7.5-15)$ & 0.421 \\
\hline Length of ICU stay (days) & $4(3-18.5)$ & $13(17.5-23.5)$ & 0.056 \\
\hline
\end{tabular}

APACHE II Acute physiological and chronic health evaluation II score; ${ }^{*} P<0.05$

In our study, the clinical manifestations of severe $M$. pneumoniae pneumonia induced ARDS were primarily dry cough, high fever, and acute respiratory failure with bilateral consolidations on radiologic examination. Respiratory failure occurred a median of 9 days (range, 3 to 15 days) after onset of symptoms, similar to the previous descriptions [3, 29]. However, these clinical features are not specific for early recognition and diagnosis of severe M. pneumoniae pneumonia. Therefore, early and precise laboratory detection of $M$. pneumoniae infection is essential to prevent deterioration. Previous methods, such as mycoplasma culture and serological tests, which may require several weeks, are not practical. As presented in our study, early definitive diagnosis is now dependent on PCR or mNGS [25], which had high specificity and sensitivity. Thus, further development of these relatively new diagnostic tools is warranted, and should be applied in cases of severe CAP induced ARDS with suspected $M$. pneumoniae infection.

Furthermore, our study found that most of our cases had pleural effusion, and PCR was positive for M. pneumoniae in pleural effusion fluid. Similar findings were also reported in a previous case report [24]. Therefore, in patients with dry cough and difficulty obtaining a lower respiratory sample, early PCR/mNGS for $M$. pneumoniae using pleural effusion fluid may be an option. As ARDS is a clinical syndrome with many different causes and may induced by some less common pathogens, mNGS is used in our ICU for early detection of possible unknown etiology, and we found that mNGS had a good value in diagnosis for M. pneumoniae in our cases. However, in most cases mNGS is more appropriate to be used for patients with unknown etiology, and may not be suitable for routine examination of some common pathogens such as ADV, RSV, and M. pneumoniae. Thus, once commercial PCR kits are available for diagnosis of M. pneumoniae infection, it is not necessary to detect $M$. pneumoniae using $\mathrm{mNGS}$ as a primary option.

In both our case series and previously reported cases of M. pneumoniae associated ARDS, deterioration of the clinical state presumably due to a significant period of inadequate antibiotic treatment $[29,30]$. In a review of severe or fatal $M$. pneumoniae pneumonia, the average duration from onset of infection to the development of respiratory failure was 11.2 days (range, 5-21 days) [29]. Chan and Miyashita et al. reported durations of 10-15 and 9.3 days, respectively, from onset to first administration of appropriate anti-mycoplasma agents $[4,5]$. The duration of an average of 9 days to change the treatment from our study was similar to the previous studies. Therefore, our management would still be considered as late intervention, and the delay as a risk factor for development of respiratory failure and ARDS. Earlier recognition of M. pneumoniae in the differential diagnosis and 
earlier initiation of appropriate antibiotics would potentially prevent $M$. pneumoniae pneumonia from progressing to ARDS.

Furthermore, more awareness is needed on the emergence of macrolide-resistant $M$. pneumoniae infection in adults [31, 32]. A previous report from our center found the rate of resistance to macrolides was $88.3 \%$ of the isolated M. pneumoniae, and all resistant strains harbored A2063G mutations. The isolated macrolide-resistant $M$. pneumoniae were resistant to erythromycin, and also showed cross-resistance to clarithromycin and azithromycin. All isolates were sensitive to tetracyclines and fluoroquinolones. Moxifloxacin was more active than ciprofloxacin and levofloxacin [33]. However, sequencing of macrolide resistance genes is not a routine test in clinical practice in our centers, and we did not perform sequencing for macrolide resistance genes in our cases. We speculated that we have the similar high rate of resistance and similar type of resistant genes in our case series. Thus, early fluoroquinolones were considered as first line treatment for $M$. pneumonia induced severe ARDS cases in adults.

A previous epidemiological study from one of our centers (Beijing Chao-Yang Hospital) that routinely screened for $M$. pneumoniae in outpatients during 2011-2016 determined that only 14 patients out of 1127 patients (1.2\%) with M. pneumoniae infection needed ICU admission [21]. However, after that study concluded, M. pneumoniae infection was only routinely screened in patients with a diagnosis of community acquired pneumonia who were hospitalized in our general ward or admitted to our ICU. During our study period, 11 of 418 severe CAP were admitted to our ICU and were diagnosed with $M$. pneumoniae pneumonia (2.3\%). Additionally, as shown in Table 1 in our study, the first LRT sample for $M$. pneumoniae was collected on an average of $11 \pm 4$ days after the onset of symptoms. The higher rate of $M$. pneumoniae pneumonia in our case series suggests that early detection for the pathogen may be needed to start an early intervention and proper treatment.

The 3 patients with mild ARDS in our study were successfully supported by HFNC and NIV without intubation. One patient with moderate ARDS was successfully supported with a combination of HFNC and awake prone positioning, which proved safe and effective in moderate ARDS patients by our team in a prospective study [34]. HFNC or NIV, combined with early prone positioning, may be a new support strategy for acute respiratory failure in $M$. pneumoniae indunced mild to moderate ARDS patients.

Although the radiologic findings showed a diffuse alveolar pattern with consolidations and the respiratory mechanics showed decreased respiratory system compliance, most intubated patients were responsive to $\mathrm{RM}$ and prone positioning during invasive ventilation, with a maximum PEEP of 11-16 (median 14) $\mathrm{cmH}_{2} \mathrm{O}$ was applied. However, two cases deteriorated to severe hypoxia despite anti-mycoplasmal therapy and invasive ventilation, eventually requiring ECMO support. In a recent case report and literature review for use of ECMO in $M$. pneumoniae associated ARDS, the mean ECMO run was $232 \mathrm{~h} / 9.68$ days [28], similar to that of our cases. The overall survival rate for 22 cases of M. pneumoniae requiring ECMO with reported outcome was $72.7 \%(16 /$ 22), demonstrating that ECMO may be safely and effectively used to treat severe ARDS caused by M. pneumoniae infection [28].

Previous reports support the hypothesis that the severity of the disease and pulmonary infiltrates may be directly correlated with the level of the individual immune response. However, in our study, we did not observe significant increases of cell or humoral immunity as demonstrated by $\mathrm{T}$ cell subset cell count or immunoglobulin levels in more severe disease. Most interestingly, we found that with appropriate respiratory support and anti-mycoplasmal therapy, all patients had a rapid clinical improvement. Therefore, no corticosteroids were given, and all patients finally recovered from ARDS without corticosteroid use. Prolonged or inappropriate use of corticosteroids may cause excess downregulation of cell-medicated immunity and result in immunosuppression, making individuals more susceptible to more severe $M$. pneumoniae infection or opportunistic infections. A recent case report revealed that $M$. pneumoniae associated ARDS had no elevated pulmonary vascular permeability, and was successfully treated using low-dose short-term hydrocortisone, suggesting that pulmonary infiltration in ARDS caused by M. pneumoniae does not match the criteria of permeability edema observed in typical ARDS [35]. Therefore, careful consideration is required when deciding whether to use high dose corticosteroid in the future cases similar to ours.

There are several limitations for our study. First, performing statistical analysis on a small sample size was prone to bias, potentially yielding spurious findings. Increasing the sample size and collecting more cases in a further study may avoid this kind of limitation. Second, this study is a retrospective study with the associated limitations on complete data collection.

\section{Conclusions}

In conclusion, early and rapid diagnosis for severe $M$. pneumoniae pneumonia with ARDS can be achieved by PCR/mNGS test in samples from lower respiratory tract or pleural effusion. In our case series, half of M. pneumoniae induced ARDS cases were adequately supported 
with HFNC or NIV and 50\% required intubation. RM and prone position were effective in $30 \%$ of intubated cases, and $20 \%$ needed ECMO support. When early antimycoplasmal therapy was given together with sufficient respiratory support, the survival rate was high with no need for corticosteroid use.

\section{Supplementary information}

Supplementary information accompanies this paper at https://doi.org/10. 1186/s12879-020-05085-5.

Additional file 1. E-Table 1. The laboratory findings for the patients with severe M. Pneumoniae pneumonia on the first day of admission.

Additional file 2. E-Table 2. The result of pleural effusion biochemistry and pleural effusion routine of the 4 severe M. pneumoniae pneumonia.

Additional file 3. E-Table 3. Cell-mediated immunity and Humoral immunity on the immunocompetent patients with severe M. Pneumoniae pneumonia on the first day of admission.

\section{Abbreviations}

ARDS: Acute respiratory distress syndrome; BALF: Broncheoalveolar lavage fluid; CXR: Chest X-ray; ECMO: Extracorporeal membrane oxygenation; HFNC: High-flow nasal cannula; HRCT: High-resolution computed tomography; ICU: Intensive care unit; IMV: Invasive mechanical ventilation; LRT: Lower respiratory tract; NIV: Non-invasive ventilation

\section{Acknowledgements}

We thanked Ann Andee Wang M.D. (from Feinberg School of Medicine, Northwestern University, Chicago, IL, USA) for her advices and help for the manuscript revision.

\section{Authors' contributions}

$L D, Y Z, X L, R W, Y L, X T, B S$ and $H H$ carried out the treatment, collected analyzed the data and wrote the manuscript. $\mathrm{HH}$ and LD conceived of the study, and participated in its design and coordination and draft the manuscript. All authors read and approved the final manuscript.

\section{Funding}

No.

\section{Availability of data and materials}

The datasets used and/or analysed during the current study are available from the corresponding author on reasonable request.

\section{Ethics approval and consent to participate}

This study is approved by the IRB of Beijing Chao-Yang Hospital and Beijing Luhe Hospital. Written informed consent was obtained from all participants (where participants are children under 16 years old, from their parent or guardian).

\section{Consent for publication}

Written informed consent was obtained from all participants for their data to be used for research and publication. Written informed consent for participation in the study was obtained where participants are children (under 16 years old) from their parent or guardian. We confirm that the patients, and in the case of minors their parents/guardians, provided written informed consent for the publication of potentially identifying images and clinical details.

\section{Competing interests}

The authors declare that no conflicts of interests.

\section{Author details}

'Department of Respiratory and Critical Care Medicine, Beijing LuHe Hospital, Capital Medical University, Beijing 101149, China. ${ }^{2}$ Department of Respiratory and Critical Care Medicine, Beijing Institute of Respiratory Medicine, Beijing Key Laboratory of Respiratory and Pulmonary Circulation, Beijing Chao-Yang
Hospital, Capital Medical University, No. 8 Gongren Tiyuchang Nanlu, Chaoyang District, Beijing 100020, China.

Received: 29 January 2020 Accepted: 12 May 2020

Published online: 24 May 2020

\section{References}

1. Cao B, Ren LL, Zhao F, Gonzalez R, Song SF, Bai L, Yin YD, Zhang YY, Liu YM, Guo P, et al. Viral and mycoplasma pneumoniae community-acquired pneumonia and novel clinical outcome evaluation in ambulatory adult patients in China. Eur J Clin Microbiol Infect Dis. 2010;29(11):1443-8.

2. Tao LL, Hu BJ, He LX, Wei L, Xie HM, Wang BQ, Li HY, Chen XH, Zhou CM, Deng WW. Etiology and antimicrobial resistance of community-acquired pneumonia in adult patients in China. Chin Med J. 2012;125(17):2967-72.

3. Izumikawa K. Clinical features of severe or fatal mycoplasma pneumoniae pneumonia. Front Microbiol. 2016;7:800.

4. Chan ED, Welsh CH. Fulminant Mycoplasma pneumoniae pneumonia. West J Med. 1995;162(2):133-42.

5. Miyashita N, Obase Y, Ouchi K, Kawasaki K, Kawai Y, Kobashi Y, Oka M. Clinical features of severe mycoplasma pneumoniae pneumonia in adults admitted to an intensive care unit. J Med Microbiol. 2007;56(Pt 12):1625-9.

6. Fischman RA, Marschall KE, Kislak JW, Greenbaum DM. Adult respiratory distress syndrome caused by mycoplasma pneumoniae. Chest. 1978;74(4): 471-3.

7. Jastremski MS. Adult respiratory distress syndrome due to mycoplasma pneumoniae. Chest. 1979;75(4):529.

8. Koletsky RJ, Weinstein AJ. Fulminant mycoplasma pneumoniae infection. Report of a fatal case, and a review of the literature. Am Rev Respir Dis. 1980;122(3):491-6.

9. Dixon C. Fulminant mycoplasma pneumoniae infection: report of a fatal case, and a review of the literature. Am Rev Respir Dis. 1981;123(2):241-2.

10. Dixon C. Mycoplasmal pneumonia and adult respiratory distress syndrome: a complication to be recognized. J Natl Med Assoc. 1981;73(6):549-52.

11. Harris LF, Swann P. Mycoplasmal pneumonia. Three severe cases of a common infection. Postgrad Med. 1984;76(6):71-2, 75, 78 passim.

12. Van Bever HP, Van Doorn JW, Demey HE. Adult respiratory distress syndrome associated with mycoplasma pneumoniae infection. Eur J Pediatr. 1992;151(3):227-8

13. Shah DC, Muthiah MM. Adult respiratory distress syndrome due to mycoplasma pneumonia. Postgrad Med J. 1996;72(846):241-2.

14. Baum H, Strubel A, Nollert J, Layh-Schmitt G. Two cases of fulminant mycoplasma pneumoniae pneumonia within 4 months. Infection. 2000; 28(3):180-3.

15. Radisic M, Torn A, Gutierrez P, Defranchi HA, Pardo P. Severe acute lung injury caused by mycoplasma pneumoniae: potential role for steroid pulses in treatment. Clin Infect Dis. 2000;31(6):1507-11.

16. Takiguchi Y, Shikama N, Aotsuka N, Koseki H, Terano T, Hirai A. Fulminant Mycoplasma pneumoniae pneumonia. Intern Med. 2001;40(4):345-8.

17. Chian CF, Chang FY. Acute respiratory distress syndrome in mycoplasma pneumonia: a case report and review. J Microbiol Immunol Infect. 1999; 32(1):52-6.

18. Daxboeck F, Eisl B, Burghuber C, Memarsadeghi M, Assadian O, Stanek G. Fatal mycoplasma pneumoniae pneumonia in a previously healthy 18-yearold girl. Wien Klin Wochenschr. 2007;119(11-12):379-84.

19. Diaz MH, Benitez AJ, Cross KE, Hicks LA, Kutty P, Bramley AM, Chappell JD, Hymas W, Patel A, Qi C, et al. Molecular detection and characterization of mycoplasma pneumoniae among patients hospitalized with communityacquired pneumonia in the United States. Open Forum Infect Dis. 2015;2(3): ofv106.

20. Khoury T, Sviri S, Rmeileh AA, Nubani A, Abutbul A, Hoss S, van Heerden PV, Bayya AE, Hidalgo-Grass C, Moses AE, et al. Increased rates of intensive care unit admission in patients with mycoplasma pneumoniae: a retrospective study. Clin Microbiol Infect. 2016;22(8):711-4.

21. Qu J, Yang C, Bao F, Chen S, Gu L, Cao B. Epidemiological characterization of respiratory tract infections caused by mycoplasma pneumoniae during epidemic and post-epidemic periods in North China, from 2011 to 2016. BMC Infect Dis. 2018;18(1):335.

22. Chaudhry R, Tabassum I, Kapoor L, Chhabra A, Sharma N, Broor S. A fulminant case of acute respiratory distress syndrome associated with mycoplasma pneumoniae infection. Indian J Pathol Microbiol. 2010;53(3): 555-7. 
23. Yew P, Farren D, Curran T, Coyle PV, McCaughey C, McGarvey L. Acute respiratory distress syndrome caused by mycoplasma pneumoniae diagnosed by polymerase chain reaction. Ulster Med J. 2012;81(1):28-9.

24. Hsieh YC, Tsao KC, Huang CG, Tong S, Winchell JM, Huang YC, Shia SH, Lai SH, Lin TY. Life-threatening pneumonia caused by macrolide-resistant mycoplasma pneumoniae. Pediatr Infect Dis J. 2012;31(2):208-9.

25. Li Y, Sun B, Tang X, Liu YL, He HY, Li XY, Wang R, Guo F, Tong ZH. Application of metagenomic next-generation sequencing for bronchoalveolar lavage diagnostics in critically ill patients. Eur J Clin Microbiol Infect Dis. 2020;39(2):369-74.

26. Kawakami N, Namkoong H, Ohata T, Sakaguchi S, Saito F, Yuki H. A fulminant case of acute respiratory distress syndrome associated with mycoplasma pneumonia treated with nasal high-flow oxygen therapy. Case Rep Crit Care. 2018;2018:1067593.

27. Malhotra P, Jindal SK. Does noninvasive ventilation work in ARDS? A case report and review of the current literature. Emerg Med J. 2005;22(10):745-6.

28. Heith CS, Hume JR, Steiner ME, Fischer GA. Fulminant mycoplasma infection requiring ECMO in a previously healthy child: case report and review. J Pediatr Intensive Care. 2018;7(2):106-9.

29. Izumikawa K, Izumikawa K, Takazono T, Kosai K, Morinaga Y, Nakamura S, Kurihara S, Imamura Y, Miyazaki T, Tsukamoto M, et al. Clinical features, risk factors and treatment of fulminant mycoplasma pneumoniae pneumonia: a review of the Japanese literature. J Infect Chemother. 2014;20(3):181-5.

30. Cummiskey AG, Reardon M. Mycoplasma pneumonia: late treatment leading to acute respiratory distress syndrome and renal failure. Eur J Case Rep Intern Med. 2019;6(2):001003.

31. Matsumoto M, Nagaoka K, Suzuki M, Konno S, Takahashi K, Takashina T, Ishiguro N, Nishimura M. An adult case of severe life-threatening mycoplasma pneumoniae pneumonia due to a macrolide-resistant strain, Japan: a case report. BMC Infect Dis. 2019;19(1):204.

32. Yang TI, Chang TH, Lu CY, Chen JM, Lee Pl, Huang LM, Chang LY. Mycoplasma pneumoniae in pediatric patients: do macrolide-resistance and/or delayed treatment matter? J Microbiol Immunol Infect. 2019;52(2): 329-35.

33. Qu J, Chen S, Bao F, Gu L, Cao B. Molecular characterization and analysis of mycoplasma pneumoniae among patients of all ages with community-acquired pneumonia during an epidemic in China. Int J Infect Dis. 2019;83:26-31.

34. Ding $L$, Wang $L, M a W, H e ~ H$. Efficacy and safety of early prone positioning combined with HFNC or NIV in moderate to severe ARDS: a multi-center prospective cohort study. Crit Care. 2020;24(1):28.

35. Takahashi N, Shinohara T, Oi R, Ota M, Toriumi S, Ogushi F. Acute respiratory distress syndrome caused by mycoplasma pneumoniae without elevated pulmonary vascular permeability: a case report. Thorac Dis. 2016;8(5):E319-24.

\section{Publisher's Note}

Springer Nature remains neutral with regard to jurisdictional claims in published maps and institutional affiliations.

Ready to submit your research? Choose BMC and benefit from:

- fast, convenient online submission

- thorough peer review by experienced researchers in your field

- rapid publication on acceptance

- support for research data, including large and complex data types

- gold Open Access which fosters wider collaboration and increased citations

- maximum visibility for your research: over $100 \mathrm{M}$ website views per year

At $\mathrm{BMC}$, research is always in progress.

Learn more biomedcentral.com/submissions 